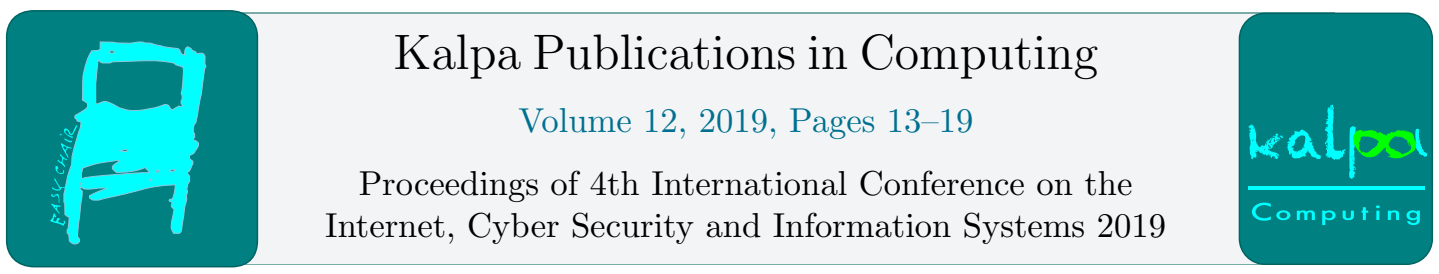

\title{
An exploratory study on telemedicine in the South African public health sector: A case of Gauteng Province
}

\author{
Tope Samuel Adeyelure ${ }^{1^{*}}$ and Billy Mathias Kalema ${ }^{2}$ \\ 1,2 Tshwane University of Technology \\ Adeyelurets@tut.ac.za, KalemaBMetut.ac.za
}

\begin{abstract}
Health systems in South African comprises of both the private sector and the public sector, run by the government. Majority of the population access health services through public clinics and hospitals. Public health sectors are understaffed, with inadequate resources, especially in rural areas. Information and communication technology and its applications can be seen as a way forward to counteract some of the challenges faced in public health sectors. Telemedicine can be tool to assist in bridging the gaps in this regard. Telemedicine is health-care delivery concept that enables distant to be examined by medical practitioners using telecommunications technologies. Therefore, this research study aimed to investigate factors that influences users' readiness to accept telemedicine in South African public health sectors environment. To achieve this objective, this study followed a positivism paradigm, supported by the quantitative research approach. Organization and Environment (in TOE framework) constructs, technology compatibility (in model of information technology implementation), perceived usefulness and perceived ease of use (in Technology Acceptance Model, (TAM))guided the development of hypotheses and their testing for this study. The findings reveal that technology, environment, organization, information quality, and security are factors that inform pre-implementation of telemedicine in South African public health sector.
\end{abstract}

\section{Introduction}

Health is a global concern that needs the attention of every nation, society and individual. According to Mahlathi and Dlamini (2015) SA has an estimated population of 54956900 , the majority of whom access health services through government-run public clinics and hospitals. Quality healthcare is considered a fundamental human right for all citizens in South Africa. The challenge remains in providing quality health care to all, especially to people living in rural areas (Colvin et al., 2011). Quality, equity and access, are key issues facing health care in both developed and economically less 
developed countries. WHO (2010)'s vision is to create and implement a health care approach that will grant the entire population access to quality health care. The term Telemedicine was first coined by Thomas Bird in 1970 and literally means "Healing at a distance" (from Greek "tele" - distance and Latin "Medicus" - medicine) (Strehle and Shabde, 2006). WHO (2010) further states that Telemedicine uses ICTs to overcome geographical barriers and increase access to health care services. Kruse et al. (2016) came to a conclusion based on there study that telemedicine has the greatest impact on access to basic health. Nair et al. (2018) further state that telemedicine is promising due to its versatility, non-invasive nature and the increasing global everyday prevalence of the Internet and communication devices.

\section{Related Work}

Walters et al. (2018) identified the design requirements for Teledermatology Scale-up Framework (TDSF). The study stated that the PHS of SA's KwaZulu-Natal province began synchronous TD in 2003, followed by spontaneous asynchronous (mobile) TD in 2013, but there was never a full scale-up formally planned or followed.

Nair et al. (2018) studied and conducted The Effectiveness of Telemedicine to Address Maternal Depression: A systematic review and meta-analysis. The appropriateness of the implementation of telemedicine is often poorly informed or uninformed. A formal analysis of telemedicine implementation could identify appropriate patients, conditions and settings, hence this study identified the gap to assess current knowledge and systematically review the evidence of effective telemedicine so as to inform future literature.

Okoroafor et al. (2017) conducted a study in Telemedicine and biomedical care in Africa: Prospects and challenges and identified the gap to critically examine the prospects and challenges of telemedical practice in Africa through a systematic review of 31 relevant publications. The study states that telemedicine holds a good prospect in improving the health conditions of the people, especially in environments with limited option of treatment such as Africa. However, the complexity of African society in the context of social structure and cultural dynamism may be an impediment to proper implementation of health care delivery through telemedical practice.

\section{Problem Statement}

The NDoH (2018) recognizes telemedicine as a priority tool in the delivery of health care as well as enhancing the knowledge of those working in the medical field. van Dyk et al. (2010) studied the Regional Innovation System for Telemedicine in SA, while Okoroafor et al. (2017) conducted and studied the prospects and challenges in telemedicine and biomedical. Aldossary et al. (2017) studied the development of a telemedicine planning framework based on needs assessment and Nwabueze et al. (2009) studied the effect of culture on telemedicine in Medically Underserved Communities. Based on the articles reviewed no study have looked at factors that informs user readiness to accept telemedicine in SA PHS.

Hence, this study will address this gap by determining factors that will influence user readiness to accept Telemedicine in South African PHS.

\section{Study Objective}

The study aim to determine factors that influences users' readiness to accept Telemedicine in South African PHS 


\section{Research Methodology}

The study used a positivism research paradigm and the research approach used is a quantitative method. Considering the research objective, the study used closed-ended questionnaires to collect data from participants. Collected data was thoroughly analyzed and interpreted using the SPSS tool. The data were collected using a cross-sectional method that is quantifiable in relation to dependent and independent variables, and to recognize the pattern of relationship from participants at one point in time; the collection of data was mastered through administration of distributed survey questionnaires to participants in and around Gauteng Province. The research model of the study informed the formulation of the questionnaire. The identified variables from the theoretical framework were used to formulate the survey questionnaire.

\section{Data Analysis}

\subsection{Descriptive Analyses}

In Table 1, five (5) constructs, (i.e. TCOMP, ENV, ORG, PU, and PEU) have a regular count over 3.60. Once all regular values are, adjusted to the closest full numeral, the values are over 4.00. It demonstrates that respondents concur that the tested variables have an effect and/or impact on preimplementation of telemedicine.

\begin{tabular}{lllllllll}
\hline & & $\mathrm{N}$ & $\begin{array}{l}\text { Minimu } \\
\mathrm{m}\end{array}$ & $\begin{array}{l}\text { Maxim } \\
\text { um }\end{array}$ & Mean & & Std. Deviation & Skewness \\
\cline { 2 - 9 } & Statistic & Statistic & Statistic & Statistic & Std. Error & Statistic & Statistic & Std. Error \\
\hline TCOMP & 130 & 3.00 & 5.00 & 4.1923 & .06651 & .75838 & -.338 & .212 \\
ENV & 130 & 1.00 & 5.00 & 3.9846 & .10417 & 1.18769 & -1.098 & .212 \\
ORG & 130 & 3.00 & 5.00 & 4.3000 & .06264 & .71420 & -.561 & .212 \\
PU & 130 & 1.00 & 5.00 & 4.0385 & .08849 & 1.00890 & -1.061 & .212 \\
PEU & 130 & 2.00 & 5.00 & 3.7346 & .06557 & .74758 & -.420 & .212 \\
$\begin{array}{l}\text { Valid N } \\
\text { (listwise }\end{array}$ & & & & & & & & \\
) & & & & & & & \\
\hline
\end{tabular}

Table 1: Descriptive Results

\subsection{Correlation Analysis Results}

The Pearson correlation analysis was, done on SPSS to examine the relationship amongst variables using coded statistics from the survey. Results in Table 2 below have a positive correlation and shows 
that all variables hold a positively high linear correlation and it appears that the variables are highly significantly correlated with each other.

\begin{tabular}{|c|c|c|c|c|c|c|c|}
\hline & & TSQ & TIQ & TSysQ & TVol & Tsec & TRtoC \\
\hline \multirow{4}{*}{ TCOM } & Pearson & 1 & & & & & \\
\hline & Correlation & 1 & & & & & \\
\hline & Sig. (2-tailed) & & & & & & \\
\hline & $\mathrm{N}$ & 130 & & & & & \\
\hline \multirow{4}{*}{ ENV } & Pearson & .708 & 1 & & & & \\
\hline & Correlation & ** & 1 & & & & \\
\hline & Sig. (2-tailed) & .000 & & & & & \\
\hline & $\mathrm{N}$ & 130 & 130 & & & & \\
\hline \multirow{3}{*}{ ORG } & $\begin{array}{r}\text { Pearson } \\
\text { Correlation }\end{array}$ & ${ }_{* *} .531$ & $.624^{* *}$ & 1 & & & \\
\hline & Sig. (2-tailed) & .000 & .000 & & & & \\
\hline & $\mathrm{N}$ & 130 & 130 & 130 & & & \\
\hline \multirow{3}{*}{ PU } & $\begin{array}{l}\text { Pearson } \\
\text { Correlation }\end{array}$ & ** .711 & $.736^{* *}$ & $.793^{* *}$ & 1 & & \\
\hline & Sig. (2-tailed) & .000 & .000 & .000 & & & \\
\hline & $\mathrm{N}$ & 130 & 130 & 130 & 130 & & \\
\hline \multirow{3}{*}{ PEU } & $\begin{array}{r}\text { Pearson } \\
\text { Correlation }\end{array}$ & ** .672 & $.827^{* *}$ & $.684^{* *}$ & $.835^{* *}$ & 1 & \\
\hline & Sig. (2-tailed) & .000 & .000 & .000 & .000 & & \\
\hline & $\mathrm{N}$ & 130 & 130 & 130 & 130 & 130 & \\
\hline
\end{tabular}

**. Correlation is significant at the 0.01 level (2-tailed).

\subsection{Regression analysis}

From the results in Table 3 below; the linear model's R-Square is 0.474 , thus the coefficient of determination is 0.474 . This means that the model developed in this study predicts $47.4 \%$ of the variation in pre-implementation of telemedicine. The regression equation that this study develops seems to be acceptable for making telemedicine pre-implementation predictions in the public health sector since it is just above $47 \%$.

\begin{tabular}{|c|c|c|c|c|c|c|c|c|c|}
\hline \multirow[b]{4}{*}{ M } & & & & \multirow{2}{*}{$\begin{array}{l}\text { St } \\
\text { d. }\end{array}$} & \multicolumn{5}{|c|}{ Change Statistics } \\
\hline & & & & & $\mathrm{R}$ & & & & \\
\hline & & & & Error & Squar & & & & $\mathrm{Si}$ \\
\hline & & & Adj & of the & e & F & & & g. F \\
\hline ode & & $\mathrm{R}$ & usted $\mathrm{R}$ & Estim & Chan & Chang & & $d$ & Chang \\
\hline 1 & $\mathrm{R}$ & Square & Square & ate & ge & $\mathrm{e}$ & $\mathrm{df1}$ & $\mathrm{f} 2$ & $\mathrm{e}$ \\
\hline \multirow[t]{3}{*}{1} & & .474 & .44 & .4 & .4 & 16 & 6 & 1 & .0 \\
\hline & 68 & & 6 & 5123 & 74 & .974 & & 13 & 00 \\
\hline & $8^{\mathrm{a}}$ & & & & & & & & \\
\hline
\end{tabular}


a. Predictors: (Constant), Data Quality, Technology, Environment, Information Quality, Organization,

Service Quality

Table 3: R-Square output

\subsection{Hypothesis Testing}

The Sig. value was tested against a threshold of 0.05 , which is if the Sig. value of a variable was found to be above 0.05 then the suggested hypotheses are rejected, whilst if the Sig. value of a variable is below 0.05 then the suggested hypothesis is accepted. The results of the hypotheses test are as illustrate in Table 4 below.

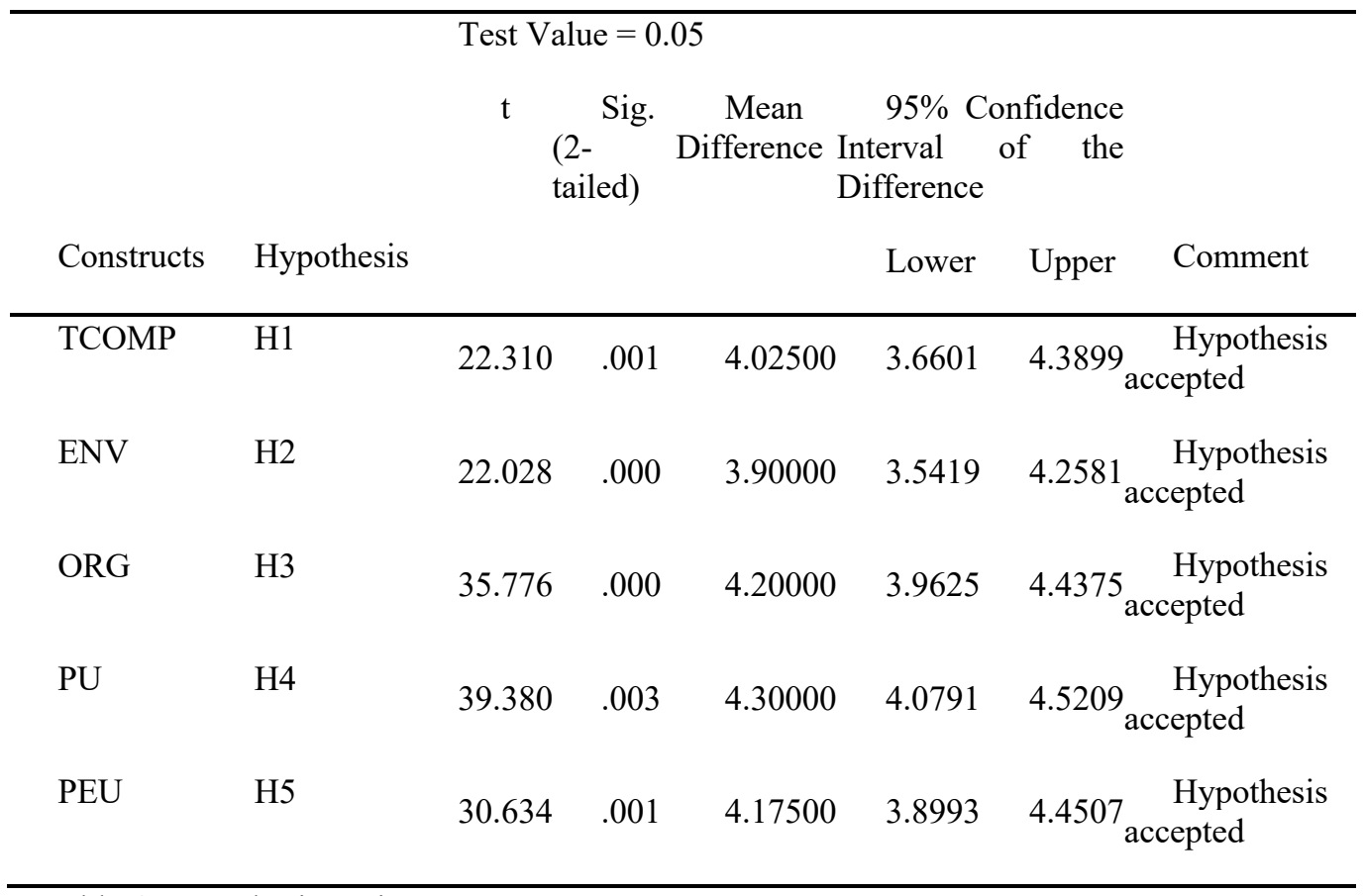

Table 4: Hypothesis testing outcome

\section{Findings and Recommendations}

Technology in the workplace allows organizations to function effectively and efficiently. However, it is essential that it is compatible with the organizational operational activities.

The result of this hypothesis is in agreement with those reported by Chen and Popovich (2003), that the technology as an imperative factor of organisational solutions. The study predicted a positive relationship between the organizational factor and user readiness to accept telemedicine. Organizational influence is a range of descriptive measures, like people, organization size, and communication methods. The study predicted a positive relationship between environmental factor and user readiness 
to accept telemedicine. Bavarsad (2013) revealed that environmental conditions like government regulations, tariffs, consumption manner are uncontrollable and influence user readiness to accept telemedicine. The uncertainty in environment influences the structuring and strategies of organizations. The greater the uncertainty the higher the chances of change in technology and this may influence user readiness to accept telemedicine. Henderson and Divett (2003), and Paul et al., (2003) ascertain the strong influence perceived usefulness has on information system acceptance by users. Increment in perceived ease of use will definitely result in user readiness to accept telemedicine. The outcome is in line with the research performed by Suki and Suki (2011), who states that perceived ease of use is an important factor in readiness to accept IT.

This study recommends validation of identified factors using a rigorous research methodology and also longitudinal time horizons may be

\section{References}

Aldossary, S., Martin-khan, M. G., Bradford, n. K., Armfield, n. R. \& Amith, a. C., 2017. The development of a telemedicine planning framework based on needs assement. Journal of medical systems, 9 .

Alsaleh, I. \& Bageel, M. 2016. Measuring user satisfaction with service quality of it department support as perceived by the users: case study of service industry.

Bavarsad, B. 2013. Studying the factors affecting the customer relations management (crm) in marun petrochemical company, interdisciplinary journal of contemporary research in business, vol 4, no 11

Chen, I.J., \& Popovich, K. 2003. "understanding customer relationship management (crm): people, process and technology", business process management journal, vol. 9 issue: 5, pp.672-688, https://doi.org/10.1108/14637150310496758

Colvin, R., Mia shim, m., Brock, D. \& Todd, G. 2011. Dermatological diagnostic acumen improves with use of a simple telemedicine system for undeserved areas of South Africa. Telemedicine and e-health, 17, 363-367.

Henderson, R. \& Divett., M., J. (2003). Perceived usefulness, ease of use and electronic supermarket use. International Journal of Human-Computer Studies, 59, 383-395

Kruse, C. S., bouffard, S., Dougherty, m. \& Parro, j. S. 2016. Telemedicine use in rural native american communities in the era of the aca: a systematic literature review. Journal of medical systems, 10.

Mahlathi, P. \& Dlamini, J. 2015. Minimum data sets for human resources for health and the surgical workforce in south africa's health system. African institute for health and leadership development, 16.

Mars, M. \& Scott, R. 2015. Spontaneous telemedicine services - what can we learn? In f. Lievens $\& \mathrm{~m}$. Jordanova (eds). Global telemedicine and ehealth updates: knowledge resources, 8, 4.

Nair, U., Armfield, N. R., Chatfield, M. D. \& Edirippulige, s. 2018. Teh effectiveness of telemedicine inteventions to address maternal depression: a systematic review and meta-analysis. Journal of telemedicine and telecare, 24, 12.

Ndoh 2018. National department of health annual report 2017/18. Rp327/2018, 216.

Nwabueze, s. N., Meso, n. P., Mengistu, K., Mbarika, V. W., Okoli, C. \& Chustz, m. 2009. The effects of culture of adoption of telemedicine in medically underserved communities. Proceedings of the 42nd hawaii international conference on system sciences, 10.

Okoroafor, I. J., Chukwuneke, F. N., Ifebunandu, N., Onyeka, T. C., Ekwueme, C. O. \& Agwuna, K. K. 2017. Telemedicine and biomedical care in africa: prospects and challenges. Nigerian journal of clinical practice, 20, 6 
Paul, L., John I., \& Pierre, C. (2003). Why do people use information technology? A critical review of the technology acceptance model. Information \& Management, 40, 191-204.

Strehle, E. M. \& Shabde, N. 2006. One hundred years of telemedicine: does this new technology have a place in paediatrics? Archives of disease in childhood,, 91, 4.

Suki, N. M and Suki, N. M. (2011) Exploring The Relationship Between Perceived usefulness, Perceived Ease Of Use, Perceived Enjoyment, Attitude And Subscribers' Intention Towards Using 3g Mobile Services. Journal of Information Technology Management. Vol. 12 (1)

Van dyk, 1., groenewald, m. \& abrahams, j. F. 2010. Towards a regional innovation system for telemedicine insouth africa. 2010 second international conference on ehealth, telemedicine, and social medicine, 4.

Walters, 1. E. M., Scott, r. E. \& Mars, m. 2018. Design requirements for a teledermatology scaleup framework. South African computer journal, 30, 33.

Who 2010. Telemedicine: opportunities and developments in member states. Global observatory for ehealth series, 2 . 